\title{
Nitride deep-ultraviolet light-emitting diodes with microlens array
}

\author{
M. Khizar, Z. Y. Fan, K. H. Kim, J. Y. Lin, and H. X. Jiang ${ }^{\text {a) }}$ \\ Department of Physics, Kansas State University, Manhattan, Kansas 66506-2601
}

(Received 5 November 2004; accepted 3 March 2005; published online 18 April 2005)

\begin{abstract}
We report on the fabrication of 280-nm AlGaN-based deep-ultraviolet light-emitting diodes (UV LEDs) on sapphire substrates with an integrated microlens array. Microlenses with a diameter of $12 \mu \mathrm{m}$ were fabricated on the sapphire substrate by resist thermal reflow and plasma dry etching. LED devices were flip-chip bonded on high thermal conductive AIN ceramic submounts to improve the thermal dissipation, and the emitted UV light was extracted through the sapphire substrates. With the integrated microlens array, a 55\% enhancement in the output power at 20-mA dc driving was achieved compared with the same LED without microlens. The light extraction enhancement is the result of the reduced internal reflections of the light caused by the microlens surface profile.
\end{abstract}

(C) 2005 American Institute of Physics. [DOI: 10.1063/1.1914960]

Ultraviolet light-emitting diodes (UV LEDs) with emission wavelengths $(\lambda)$ below $340 \mathrm{~nm}$ have many important applications, including biological and chemical agent detection, high-density data storage, and air-water purification and medical usage. In particular, an interest has developed concerning the use of compact deep-UV light source $(\lambda$ $<300 \mathrm{~nm}$ ) to increase the sensitivity of biosensors. For example, the 280-nm emission matches the absorption maxima of the naturally fluorescent amino acids tryptophan and tyrosine and is widely used as a probe of protein structure and dynamics. Though the output power of deep-UV LEDs based on nitride materials continues to increase, ${ }^{1-3}$ the emitted powers and efficiencies of nearly all the devices were significantly lower than those of the visible or the near-UV LEDs. ${ }^{4}$ It is therefore necessary to further improve the light-output efficiency and performance of deep-UV LEDs. The LED efficiency depends on the internal quantum efficiency (QE) and the light extraction efficiency. The factors responsible for the low power output, low QE, and long-wavelength emission components in deep-UV LEDs include dislocations and defects, low QE of injected carriers, barrier leakage, and device heating. ${ }^{5}$ Continuing improvement of the material quality and device structure is critical for the device performance. On the other hand, enhancing the extraction efficiency is also a critical issue, considering that with a refractive index of 2.5, a planar GaN LED structure only has a light extraction efficiency of around $5 \%{ }^{6,7}$

To reduce the problem of light trapping in the high index semiconductor, several schemes/architectures have been employed to enhance the light extraction. These include the incorporation of photonic crystals, ${ }^{8,9}$ surface roughening or textured semiconductor surface, ${ }^{10-12}$ and shaping of LED dies. ${ }^{6}$ However, many of these techniques are applied to the LED dies and are not fully compatible with the flip-chip architecture that is often needed for LEDs grown on sapphire substrates for enhanced thermal management. Here we demonstrate that integrated sapphire substrate microlens in conjunction with flip-chip bonding can be used to improve the light extraction. Microlens has been widely used for light collimation and coupling in optical systems. ${ }^{13,14}$ In recent years, monolithically integrated microlenses fabricated on

\footnotetext{
${ }^{a)}$ Electronic mail: jiang@phys.ksu.edu
}

the GaN and AlN surfaces or its sapphire substrate surface have also been reported for similar purposes. ${ }^{15-17}$ In this Letter, we report the flip-chip bonded 280-nm UV LED with integrated sapphire substrate microlens array, and the results demonstrate that microlens array is also an efficient method to enhance the light extraction for UV or deep-UV LEDs.

The UV LED structure was deposited on the basal plane sapphire substrate by metalorganic chemical vapor deposition (MOCVD). A high quality AIN epilayer with a thickness of about $1 \mu \mathrm{m}$ was grown as the epitaxial template for the subsequent device layers. Above the AlN epilayer, a 1.5$\mu \mathrm{m}$ Si-doped $n$ - $\mathrm{Al}_{0.6} \mathrm{Ga}_{0.4} \mathrm{~N}$ was grown as the $n$-type cladding layer. Following is the AlGaN-based quantum well (QW) active region. Since it is difficult to achieve a reasonable hole density in an AlGaN alloy with high Al composi-
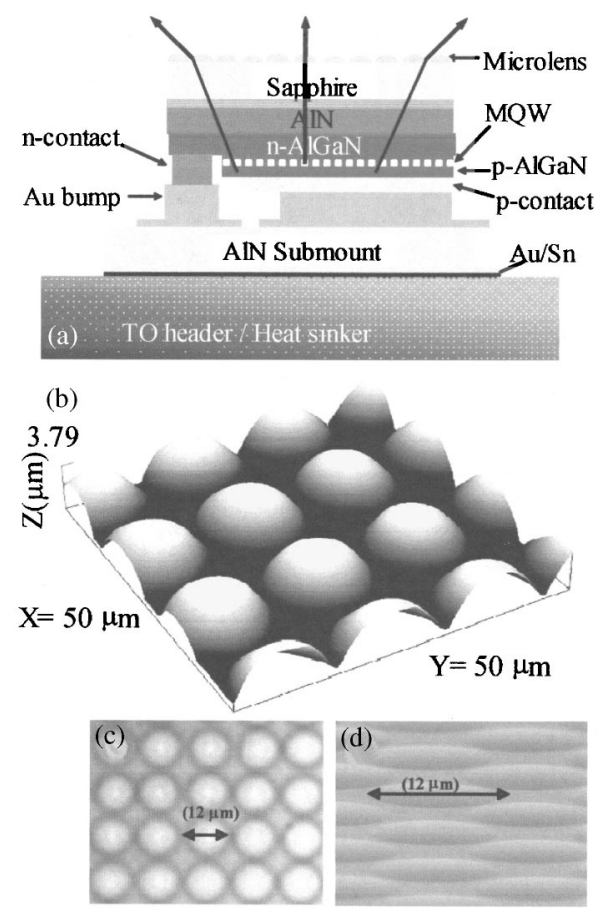

FIG. 1. (a) Schematic diagram of the flip-chip bonded deep-UV LED structure with an integrated microlens array on the sapphire substrate. (b) AFM image of photoresist after reflow. (c) Optical microscopy image and (d) SEM image of a fabricated sapphire microlens array. 


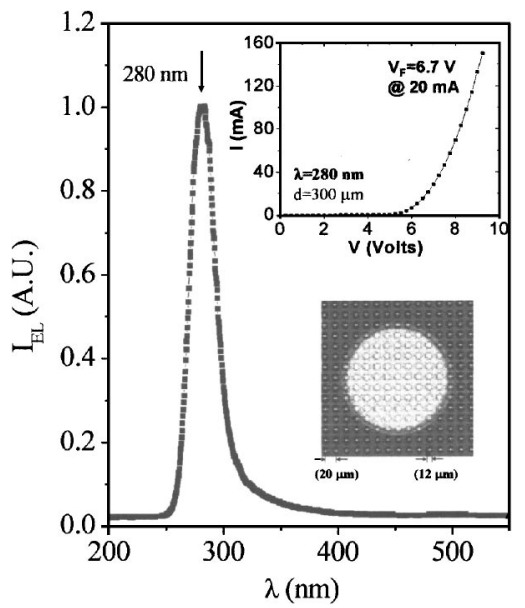

FIG. 2. The EL spectrum of a 280-nm flip-chip bonded deep-UV LED with an integrated microlens array. The inset shows the optical microscopy image of the same circular LED taken from sapphire side and its $I-V$ characteristics. Under a $20-\mathrm{mA}$ dc driving, the output power of our 280-nm disk UV LEDs with a diameter of $275 \mu \mathrm{m}$ fabricated from different wafers varies between 0.2 and $0.35 \mathrm{~mW}$.

tion, a $p-\mathrm{Al}_{0.7} \mathrm{Ga}_{0.3} \mathrm{~N}$ layer $(10 \mathrm{~nm})$ was employed as an electron blocking layer to effectively block the electron overflow, thereby enhancing the electron-hole radiative combination in the QWs. The structure was then completed with a $60-\AA$ Mg-doped $\mathrm{Al}_{0.6} \mathrm{Ga}_{0.4} \mathrm{~N}$ graded layer and 150 -nm heavily $\mathrm{Mg}$ doped $\mathrm{GaN}$ as the $p$-contact layer. Small-dimension circular LED with diameter $<300 \mu \mathrm{m}$ has a better current spreading as well as small thermal resistance. Large-size LEDs with interdigital shape are also fabricated to overcome the current crowding effect. The fabrication started from the mesa etching to expose the $n-\mathrm{Al}_{0.6} \mathrm{Ga}_{0.4} \mathrm{~N}$, followed by $\mathrm{Ti} / \mathrm{Al}$ metal deposition for the $n$ contact and $\mathrm{Ni} / \mathrm{Au}$ for $p$ contact with rapid thermal annealing.

To avoid the optical absorption by the multilayers on the top and the wire bonding pads, the emitted light from a deep-UV LED grown on the transparent sapphire substrate is commonly extracted through the AIN layer and sapphire substrate by employing flip-chip bonding. As shown in Fig. 1(a), the processed LED was flip-chip bonded with Au bumps onto a ceramic AlN submount, and finally mounted on to a TO header and heat sink for the measurement.

Flip-chip bonded LEDs allow light extraction from a nitride semiconductor ( $n \approx 2.5$ for $\mathrm{GaN}$ ) into the sapphire substrate $(n \approx 1.76)$ instead of into air, and an increase in extraction efficiency can be expected. However, there is still a large step change in the index of refraction at the interface between the sapphire substrate and the air, which causes the light to reflect back into sapphire and LED. A dome-shaped epoxylike encapsulation with a typical refractive index of 1.8 closely matched to that of sapphire should be able to solve this problem. Unfortunately, no encapsulation material that is transparent at wavelengths below $300 \mathrm{~nm}$ is available at this time. On the other hand, if the sapphire substrate is etched into a hemispherical dome, the light extracted into the sapphire substrate can get out immediately without encountering the reflection. Furthermore, the surface modification will be made on the sapphire substrate, which is well suited for the flip-chip bonding. Based on this idea, as schematically illustrated in Fig. 1(a), we fabricated a microlens array on the sapphire substrate to enhance the light extraction. sapphire substrate to enhance the light extraction. angle and multiple reflection.
Downloaded 12 Jul 2010 to 129.118.86.59. Redistribution subject to AIP license or copyright;

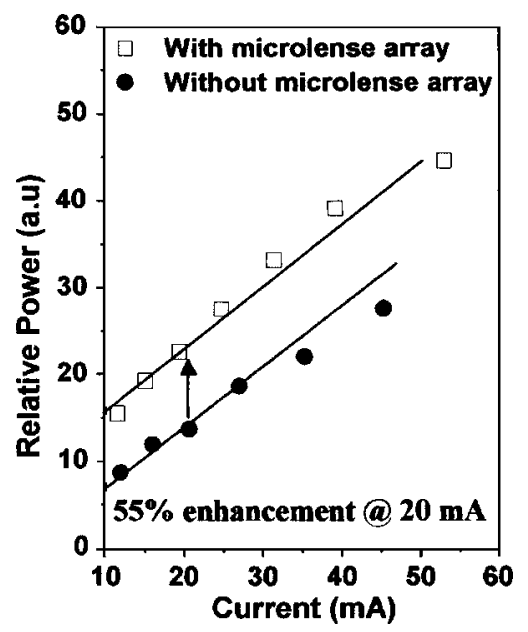

FIG. 3. Comparison of the deep-UV LED output power with and without the microlens array. A $55 \%$ increase in the optical output power by the microlens array formation was measured at $20-\mathrm{mA}$ dc driving.

Photoresist reflow and dry etching method was used for processing microlens arrays on sapphire substrates. ${ }^{10,11}$ In this method, a thick photoresist layer was spun onto the sapphire substrate and patterned by standard photolithography into cylindrical posts. After baking, the surface tension causes them to take up hemispherical shapes, shown by the atomic force microscope (AFM) image in Fig. 1(b). The preformed resist is then used as a mask for pattern transfer by inductively coupled plasma (ICP) dry etching with chlorinebased plasma chemistry. An etch rate of $130 \mathrm{~nm} / \mathrm{min}$ for sapphire was achieved. The optical microscope image [Fig. $1(\mathrm{c})$ ] and the scanning electron microscope (SEM) image [Fig. 1(d)] show the fabricated microlens array on the sapphire substrate. The fabricated microlens has a bottom diameter of $12 \mu \mathrm{m}$ and a height of $1 \mu \mathrm{m}$ with a pitch of $20 \mu \mathrm{m}$. We expect that microlenses with smaller diameters will provide a better performance due to the more pronounced surface curvature. However, it is technically more difficult to fabricate microlenses with small diameters. So $12-\mu \mathrm{m}$ diam was chosen for the ease of fabrication as well as for an observable effect.
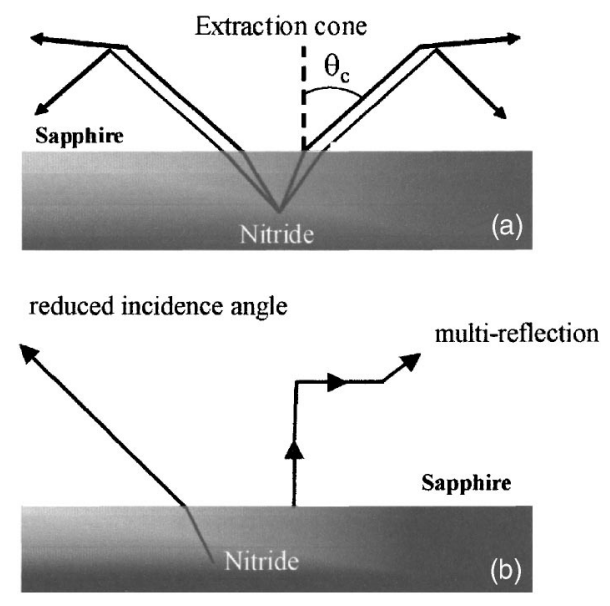

FIG. 4. Schematic diagrams to illustrate the light extraction enhancement by the microlens array formation. In (a), the planar interface limits the light extraction in a cone with most of the light entrapped in the wave-guided sapphire slab and nitride layers, while the curvature interface formed by the microlens array in (b) enhances the light extraction by reducing the incident 
The electroluminescence (EL) spectrum of a 280-nm flip-chip bonded disk UV LED is shown in Fig. 2, along with its optical microscope image and current-voltage $(I-V)$ characteristics. Under a 20-mA dc driving, the output power of our 280-nm disk UV LEDs with a diameter of $275 \mu \mathrm{m}$ fabricated from different wafers varies between 0.2 and $0.35 \mathrm{~mW}$.

To study the effect of microlens array, two representative devices diced from the nearby locations from the same processed wafer with/without microlens formation are characterized and compared with each other. The angular distribution of light measurement shows that the light-emission pattern from the sapphire substrate has no significant difference with and without microlens array. This is understandable since the microlens has a size much smaller than the LED dimension. The change in the directionality of the LED emission caused by individual microlenses is smeared out. ${ }^{17}$ To compare the light extraction efficiency, the light emission was measured with a UV-enhanced Si detector on top of the LED with a fixed distance, and the relative emission power of the LED with and without microlens array is plotted in Fig. 3. The results clearly show that the LED with microlens array produces more light output than that of without microlens. Specifically, a 55\% increase in the output power is measured at $20-\mathrm{mA}$ dc current driving. The enhanced light extraction is attributed to the curvature surface profile of the microlenses. As illustrated in Fig. 4(a), with a refractive index of 1.76, sapphire has a critical angle of around $34.6^{\circ}$; the incident light with a larger angle will be guided and trapped in the sapphire slab and part of this light will be absorbed. With the integrated microlens array, the sapphire-air interface now has a certain curvature, as shown in Fig. 4(b). The original light ray with a large incidence angle at the flat surface now has a reduced incident angle at the curved surface and is able to get out. Moreover, because of the curvature, the reflected light ray from the microlens surface also has a better chance to finally get out. As a result, more light can be extracted from the LED by the microlens array formation.

In summary, monolithic integrated arrays of microlens with a height of $1 \mu \mathrm{m}$ on the sapphire substrates of deep UV LEDs were fabricated. The output powers of the LEDs with and without microlens array were measured and compared with each other. The microlens array formation enhanced the UV LED output power by $55 \%$ at $20-\mathrm{mA}$ de driving. Thus, monolithic integrated microlens array on sapphire can be further developed and used to improve the overall efficiency of nitride-based deep-UV optoelectronic devices without the use of an external optics.

The authors wish to acknowledge support by grants from DARPA, ARO, DOE, and NSF.

${ }^{1}$ W. H. Sun, J. P. Zhang, V. Adivarahan, A. Chitnis, M. Shatalov, S. Wu, V. Mandavilli, J. W. Yang, and M. A. Khan, Appl. Phys. Lett. 85, 531 (2004).

${ }^{2}$ A. J. Fischer, A. A. Allerman, M. H. Crawford, K. H. A. Bogart, S. R. Lee, R. J. Kaplar, W. W. Chow, S. R. Kurtz, K. W. Fullmer, and J. J. Figiel, Appl. Phys. Lett. 84, 3394 (2004).

${ }^{3}$ K. H. Kim, Z. Y. Fan, M. Khizar, M. L. Nakarmi, J. Y. Lin, and H. X. Jiang, Appl. Phys. Lett. 85, 4777 (2004).

${ }^{4}$ S. Nakamura and G. Fasol, The Blue Laser Diode (Springer, New York, 1997).

${ }^{5}$ V. Adivarahan, S. Wu, J. P. Zhang, A. Chitnis, M. Shatalov, and V. Mandavilli, Appl. Phys. Lett. 84, 4762 (2004).

${ }^{6}$ A. Bergh, M. G. Craford, A. Duggal, and R. Haitz, Phys. Today 54, 42 (2001).

${ }^{7}$ E. F. Schubert, Light-Emitting Diodes (Cambrige University Press, New York, 2003).

${ }^{8}$ T. N. Oder, K. H. Kim, J. Y. Lin, and H. X. Jiang, Appl. Phys. Lett. 84, 466 (2004).

${ }^{9}$ J. Shakya, K. H. Kim, J. Y. Lin, and H. X. Jiang, Appl. Phys. Lett. 85, 142 (2004).

${ }^{10}$ I. Schnitzer, E. Yablonovitch, C. Caneau, T. J. Gmitter, and A. Scherer, Appl. Phys. Lett. 63, 2174 (1993).

${ }^{11}$ R. Windisch, C. Rooman, S. Meinlschmidt, P. Kiesel, D. Zipperer, G. H. Döhler, B. Dutta, M. Kuijk, G. Borghs, and P. Heremans, Appl. Phys. Lett. 79, 2315 (2001).

${ }^{12}$ T. Fujii, Y. Gao, R. Sharma, E. L. Hu, S. P. DenBaars, and S. Nakamura, Appl. Phys. Lett. 84, 855 (2004).

${ }^{13}$ H. P. Herzig, Mico-Optice (Taylor and Francis, London, 1997).

${ }^{14}$ A. W. Lohmann, Appl. Opt. 28, 4996 (1989).

${ }^{15}$ S. H. Park, H. Jeon, Y. J. Sung, and G. Y. Yeom, Appl. Opt. 40, 3698 (2001).

${ }^{16}$ T. N. Order, J. Shakya, J. Y. Lin, and H. X. Jiang, Appl. Phys. Lett. 82, 3692 (2003).

${ }^{17}$ H. W. Choi, C. Liu, E. Gu, G. McConnell, J. M. Girkin, I. M. Watson, and M. D. Dawson, Appl. Phys. Lett. 84, 2253 (2004). 